Atomic Energy Levels as derived from the Analyses of Optical Spectra

Vol. 1, Section 1: The Spectra of Hydrogen, Deuterium, Helium, Lithium, Beryllium, Boron, Carbon, Nitrogen, Oxygen and Fluorine. By Charlotte E. Moore. (Circular of the National Bureau of Standards, 467.) Pp. iii+75. (Washington, D.C. : Government Printing Office, 1948.) 50 cents.

THIS is apparently the first of a series of reports from the National Bureau of Standards, Wash. ington, and covers the elements of the first row of the Periodic Table. That very valuable book, "Atomic Energy States", by Bacher and Goudsmit, is now sixteen years out of date, and as it is not being revised this new series will eventually replace it.

The general arrangement follows closely that of Bacher and Goudsmit; but it is an improvement to have all spectra treated similarly with the groundstate of the atom taken as zero energy. This part is right up to date, including work up to late in 1947. The recent work of Edlén is fully treated, and many of his hitherto unpublished results are included. It is interesting to see the inclusion of the ${ }^{8} S$ level of C I at $33735 \mathrm{em}^{-1}$; this has been the subject of much discussion in connexion with the energies of carbon bonds. The introduction to the series has not yet been published, and that is quite understandable; but it is a pity that a brief key is not given to the mysterious symbols (T., G.D., etc.) which appear after the references. The present circular does not contain any diagrams of term schemes; but charts of predicted terms in the spectra of some isoelectronic sequences are promised later. This work of compiling atomic energy-levels is an extremely valuable contribution to science, and these volumes will be indispensable to anyone who uses spectroscopic data.

A. G. G.

\section{Finite Differences and Difference Equations in the Real Domain}

By Prof. Tomlinson Fort. Pp. viii +251 . (Oxford: Clarendon Press; London : Oxford University Press, 1948.) 25s. net.

$\mathrm{T}^{\mathrm{N}}$ his preface, the author states that this volume includes material for a course in finite differences as well as a treatment of a number of special topics. A selection of material was necessary in order to bring the work within reasonable compass, and also to follow somewhat the special interests of the author. The very large theory of difference equations in the field of analytic functions of a complex variable has been completely omitted. This has carried with it the omission of a variety of related subjects, particularly in infinite series.

The first six chapters constitute a readable account of the applications of the finite calculus to differencing, summation, the polynomials of Bernoulli and Euler, numerical differentiation and integration, and interpolation. The remaining eleven chapters are mainly devoted to a study of the linear recurrence relation, that is to say, the linear difference equation in which the independent variable assumes integral values. It is in this topic that the author's main interest clearly lies, but the powerful and elegant operational methods which are available in this field are barely touched. On the other hand, there are interesting and useful accounts of the linear recurrence relations of the first and second orders with periodic coefficients.

L. M. M.-T.
A Bibliography of Chemical Research in South Africa, $1910-1939$

Compiled by Dr. Herbert Coblans. (Published for the Natal University College.) Pp. ix +25 . (Cape Town : African Bookman, 1947.) 7s. 6 d.

THE author of this slender volume explains that the lack of bibliographical tools is a real handicap to research workers in South Africa, since the proceedings of the learned societies there have no cumulative indexes. Many of the papers listed, however, have been published in European or American journals, and chemical research is perhaps one of the best abstracted fields of work. The information given can, therefore, be of special interest only to those chemists who wish to know which publications were by South African authors. Full titles of the papers are given and the references to the publications. The classification is according to subjects in decimal order based on the general principle of increasing specialization. In each section, the order is according to year of publication. Dr. Coblans is himself a chemist, and the accuracy of the book may, therefore, be taken for granted. His position as librarian of Natal University College vouches for his bibliographical ability. It is clear from the contents of the book that chemists in South Africa are making notable contributions to research in their subject, as was, in fact, generally realized before its publication.

\section{Studies and Essays presented to R. Courant on} his 60th Birthday, January 8, 1948

Pp. viii +470 . (New York and London: Interscience Publishers, Inc., 1948.) $33 s$.

DROF. R. COURANT, formerly of Göttingen and now of New York, who is known to all mathematicians by Courant and Hilbert's "Methoden der Mathematischen Physik", attained his sixtieth birthday on January 8, 1948. To commemorate the occasion a number of his friends and colleagues were invited to contribute articles for a volume to be presented to him. Among the thirty-eight mathematicians who responded to this invitation are included such well-known authorities as Harald Bohr, Carathéodory, Franck, Hadamard, Kramers, Neugebauer, Pólya, and Van der Waerden. The papers cover $a$ wide field, corresponding to the wide range of Courant's own work, and some of them are direct developments from this work. Although most of the authors are foreign, all but six of the papers are in English.

The Cathode Ray Oscillograph in Industry

By Dr. W. Wilson. Third edition, revised. Pp. xii+252. (London: Chapman and Hall, Ltd., 1948.) 18s. net.

BECAUSE of demand the present text has been B off the market for some time, so the author has taken the opportunity of bringing some of the matter up to date and also of incorporating applications of cathode-ray tubes which have been recently released. Not only would radar have been impossible but also many of the measurements and controls of industry would have been impracticable without the cathoderay tube. It has become a tool of precision and a means of display. The types available and applicable to special tasks in industry are so well treated by the author that this edition will also soon be disposed of.

L. E. C. H 